Colman, A. M. (1998). Modelling imitation with sequential games. Behavioral and Brain Sciences, 21, 686.

\title{
Modelling imitation with sequential games
}

Andrew M. Colman

Department of Psychology, University of Leicester, Leicester LE1 7RH, England. amc@leicester.ac.uk; www.le.ac.uk/psychology/amc/amc.html

\begin{abstract}
A significant increase in the probability of an action resulting from observing that action performed by another agent cannot, on its own, provide persuasive evidence of imitation. Simple models of social influence based on two-person sequential games suggest that both imitation and pseudo-imitation can be explained by a process more fundamental than priming, namely, subjective utility maximization.
\end{abstract}

Imitation has been operationally defined as a significant increase in the probability of an action as a result of observing of another agent performing that action. Byrne \& Russon (B\&R) have argued persuasively that this interpretation can lead to behavior being defined as imitative when it can be explained more simply by priming. I shall argue that such behavior can be explained even more simply by subjective utility maximization and that this need not necessarily rule out imitation.

Two-person game theory is applicable to any social interaction involving two agents (players), each choosing between two or more ways of acting (strategies), the outcome depending on the choices of both agents and the agents having well-defined preferences among the possible outcomes. Imitation, impersonation, emulation, and observational learning can all be modelled by two-person games. Potential strategies range from simple behavioral acts such as tongue protrusion by a mother, possibly inducing action level imitation by an infant, to complex behavioral sequences such as the removal of stings from nettles by a mountain gorilla, possibly inducing program level imitation by a conspecific. Preferences are expressed as payoffs representing units of subjective utility associated with maternal pride, satisfaction of hunger, social approval, or, more generally, any form of reinforcement or (in evolutionary games) change in Darwinian fitness. The fundamental game-theoretic assumption is that players are motivated solely to maximize their own individual payoffs. [See also Maynard Smith: “Game Theory Without Rationality”, BBS, 7(1), 1984.]

There is a large class of games in which players end up choosing the same strategy. The strategy sets from which they choose may be large, but for simplicity consider a familiar twostrategy example, the Prisoner's Dilemma game:

$\begin{array}{ccl} & C & D \\ C & (3,3) & (1,4) \\ D & (4,1) & (2,2)\end{array}$

One player chooses column $C$ (cooperate) or $D$ (defect), the other player row $C$ or $D$, and for each possible strategy combination, the pair of numbers in the payoff matrix indicates the (ordinal-level) payoffs to the row-chooser and the column-chooser, respectively. The experimental literature on this game includes numerous studies of human behavior (reviewed by Colman 1995, pp. 134-185) and some of nonhuman behavior (e.g., Flood et al. 1983; Gardner et al. 1984). The evidence confirms that a row-chooser who has reason to expect the column-chooser to defect usually also defects, and this makes sense because if the column- 
chooser opts for $D$, then the row-chooser receives a higher payoff by also opting for $D$ (2 units) than by opting for $C$ (1 unit). It follows that in a sequential version of the game in which the column-chooser moves first and opts for $D$, the row-chooser, moving second with perfect information of the column-chooser's strategy, is almost certain to follow suit.

Should such behavioral convergence be interpreted as imitation? I believe it should not, for the following reason. The row-chooser selects the same strategy as the column-chooser but does not select it because it is the same. The strategic structure of the game encourages the rowplayer to defect irrespective of the action of the column-chooser, because the defecting strategy is dominant in the sense that it yields a better payoff to the row-chooser irrespective of the column-chooser's strategy. Consequently, in the sequential version of the game, a row-chooser who responds to a column-chooser's defecting strategy by following suit cannot validly be described as imitating the column-chooser's action, because defection is mandated by selfinterest in any case.

Interestingly, this objection does not apply to all strategic interactions. Consider next the Stag Hunt game (Lewis 1969):

$\begin{array}{ccc} & C & D \\ C & (3,3) & (1,2) \\ D & (2,1) & (2,2)\end{array}$

Here again, a self-interested player will seek to choose the same strategy as the co-player, but in this case the strategy will be chosen precisely because it is the same as the co-player's. The row-chooser will defect if there is reason to expect the column-chooser to defect but will cooperate if there is reason to expect the column-chooser to cooperate. Thus, in a sequential version of this game, the row-chooser, moving second, will respond to $D$ with $D$ to receive a payoff of 2 rather than 1 and will respond to $C$ with $C$ to receive a payoff of 3 rather than 2 . The players' choices will tend to converge, as in the Prisoner's Dilemma game, but in the sequential version of this game it seems reasonable to interpret such behavioral convergence as imitative, because the row-chooser's strategy is directly induced by the column-chooser's.

Byrne \& Russon (B\&R) may reject this as a model of imitation because it is explicable by the more fundamental process of utility maximization (and also perhaps by priming). This objection could be dismissed on the ground that all voluntary behavior involves choice and all choice can be interpreted in terms of maximizing subjective expected utility (Jeffrey 1983; Kahneman \& Tversky 1979). B\&R would certainly reject it in cases in which the strategies represent non-novel forms of behavior, though the model is neutral on this point. The counterargument to this is that an action directly induced by observing the same action being performed by another agent may be imitative even if it lacks novelty. A teenager who is induced to wear an old pair of jeans by observing an admired role model wearing jeans is evidently imitating the role model, and any interpretation of imitation that excludes such a case seems perverse and at odds with the conventional meaning of the concept.

What is the essential structural difference between games such as the Prisoner's Dilemma and those such as the Stag Hunt, which allow strategic convergence to be interpreted as imitation in the latter class but not the former? The answer relates to Nash equilibria. A Nash equilibrium is a combination of strategies that are best responses to each other. In the Prisoner's Dilemma game, the best response to $C$ is $D$ and the best response to $D$ is also $D$, hence the only Nash equilibrium in the game is the strategy combination $D D$ in the lower-right cell, yielding payoffs of 2 units to each player. Consequently, in the sequential version of this game, the best response of the row-chooser, moving second and pursuing self-interest, is $D$ irrespective of the 
column-chooser's strategy. However, in the Stag Hunt game, the best response to $C$ is $C$ and the best response to $D$ is $D$, yielding two Nash equilibria in the top-left and bottom-right cells, and these two equilibria correspond to different strategies of the row-chooser. It seems reasonable to define an action as imitative if it results from observing another agent making the same voluntary choice and is directly induced by the other agent's choice, as in games belonging to the Stag Hunt class.

In some sequential games, an action may be directly induced by another agent's voluntary choice but may nevertheless fail to satisfy this definition of imitation. Consider, for example, the Hawk-Dove game (Maynard Smith \& Price 1973):

$\begin{array}{ccl} & C & D \\ C & (3,3) & (2,4) \\ D & (4,2) & (1,1)\end{array}$

In this game there are two asymmetric Nash equilibria. The best response to $C$ is $D$, and the best response to $D$ is $C$, hence the bottom-left and top-right cells represent Nash equilibria. In a sequential version of this game, although the strategy of the row-chooser, moving second, is induced by the action of the column-chooser who moves first, it does not satisfy my definition of imitation, because the behavior that is induced differs from the behavior that induces it. There are many other games of this type in which the players, by acting to maximize their individual self-interests, end up choosing different strategies.

\section{ACKNOWLEDGMENT}

Preparation of this article was facilitated by Grant No. L122251002 from the Economic and Social Research Council of the U.K. as part of the research program on Economic Beliefs and Behavior.

\section{References}

Colman, A. M. (1995). Game theory and its applications in the social and biological sciences (2nd ed.). Routledge.

Flood, M. M., Lendenmann, K., \& Rapoport, A. (1983). 2 x 2 games played by rats: Different delays of reinforcement as payoffs. Behavioral Science, 28, 65-78.

Gardner, R. M., Corbin, T. L., Beltramo, J. S., \& Nickell, G. G. (1984). The Prisoner's Dilemma Game and cooperation in the rat. Psychological Reports, 55, 687-696.

Jeffrey, R. C. (1983). The logic of decision (2nd ed.). University of Chicago Press.

Kahneman, D. \& Tversky, A. (1979). Prospect theory: An analysis of decision under risk. Econometrica, 47, 263-291.

Lewis, D. K. (1969). Convention: A philosophical study. Harvard University Press.

Maynard Smith, J. \& Price, G. R. (1973). The logic of animal conflict. Nature, 246, 15-18. 